\title{
Elastic response and wrinkling onset of curved elastic membranes subjected to indentation test
}

\author{
R. Bernal ${ }^{1}$, Ch. Tassius ${ }^{2}$, F. Melo ${ }^{1}$, and J-Ch. Géminard ${ }^{2}$ \\ 1 Departamento de Física, Universidad de Santiago de Chile, Av. Ecuador 3493 Estación Central, Santiago Chile \\ 2 Université de Lyon, CNRS - 46 Allée d'Italie, 69364 Lyon cedex 07 France.
}

Received: date / Revised version: date

\begin{abstract}
Starting from a polymeric-fluid droplet, by vulcanization of the fluid free surface, curved elastic membranes, several nanometers thick and a few millimeters in diameter, which enclose a constant fluid volume, are produced. In an indentation-type test, carried out by pushing the membrane along its normal by means of a microneedle, under some conditions, wrinkles are likely to appear around the contact region. Interestingly, we observe that the instability does not significantly alter the force-displacement relation: the relation between the force and the displacement remains linear and the associated stiffness is simply proportional to the tension of the membrane. In addition, we determine that the wrinkles develop when the stretching modulus of the membrane compares with its tension, which provides a useful method to estimate the elastic constant.
\end{abstract}

PACS. $46.32 .+\mathrm{x}$ Static buckling and instability -81.70. Bt Mechanical testing, impact tests, static and dynamic loads - 68.60.Bs Mechanical and acoustical properties.

\section{Introduction}

The mechanical properties of thin elastic membranes are of wide interest to control processes such as cell motility $[1,2]$, wound healing [3], electronic surface protection [4] and fabrication and characterization of ordered structures at the nano and micro scale $[5,6]$. For instance, in biological applications, it has been recently shown that hollow structures, like vesicles or capsules, have high potential for holding sensitive contents until release into the external environment is appropriate $[7,8]$. The properties and structure of these capsules are assessed with indentationtype tests by deforming them axi-symmetrically with the help of microcantilevers or flat surfaces. Relations between the indentation force, the membrane inner pressure and the structure deflection have been obtained $[7,9]$. However, due to large membrane deflection involved in such tests, wrinkling is often observed when the (compressive) stress exceeds a certain threshold $[10,11,12]$. In turn wrinkles structures might reveal important information on membrane properties and thickness as well $[12,13,14]$.

Here we develop further the potential of indentation tests for the assessment of mechanical properties of thin curved membranes. Our configuration has the advantage of providing well-defined boundary-conditions, which allows us to develop simple numeric description accounting for large deflections of the membrane. We observe that the applied force depends linearly on the imposed displacement and, from the theoretical and numerical analysis of the problem, we show that the stiffness relates to
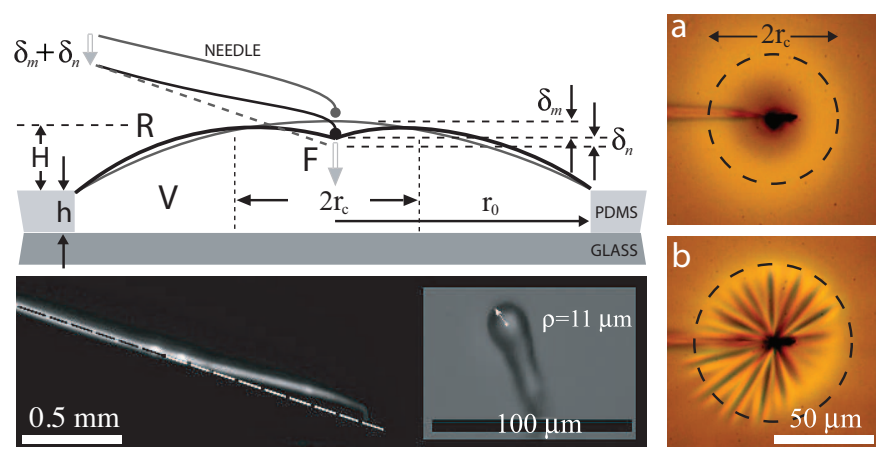

Fig. 1. Sketch of the sample.

Top: Schematic of force measurement indicating the deflection, $\delta_{n}$ and the indentation $\delta_{m}$. Bottom: The needle tip shape designed to avoid off center contact. Right panel a) A concave central part marked by a brighter ring; $H / r o=0.23$. Right panel b) Radial wrinkles for $H / r o=0.40$.

the membrane tension. In addition, we observe that radial wrinkles, which are likely to form around the indenter, do not significantly affect the force-displacement relation. We determine that the onset of the instability depends on the membrane tension, wrinkles forming when the tension compares with the stretching modulus of the membrane. Thus, measurements of the tension, thanks to the indentation test, and the determination of the instability onset provides, in a single experiment, a useful method to estimate the membrane elastic modulus. 


\section{Experimental procedure}

The sample consists of a curved thin membrane attached to the upper circular edge of a cylindrical rigid cavity (Fig. 1, Top). First a homogeneous substrate is obtained by curing a layer (thickness $h=500 \mu \mathrm{m}$ ) of poly dimethyl siloxane (PDMS, Sylgard 184), previously deposited onto a piece of glass plate. The cylindrical cavity is then created by cutting a hole of diameter $2 r_{0}=3 \mathrm{~mm}$ in the substrate.

To obtain the elastic membrane, a given volume $V$ of PDMS polymer fluid is injected inside the cavity obtaining a drop whose free surface has a given curvature. Drop curvature is easily increased by increasing the volume $V$. Membranes are prepared by vulcanization, as described in Refs. [1,2], of the surface of the polymeric fluid. Fluid vulcanization is simply produced by the action of a homogeneous flame from a Bunzer-type burner $[1,2,15]$. During this process, and in order to obtain a more homogeneous film, the sample is quickly turned upside-down and displaced at constant horizontal velocity (a few $\mathrm{mm} / \mathrm{s}$ ) just above the blue part of the flame. The flame width being about $5 \mathrm{~mm}$ in our case, the contact-time between the flame and the fluid surface is about $1 \mathrm{~s}$ which insures homogeneous membrane thickness at large scale. Thus, in our experimental conditions, we produce a membrane of reproducible thickness $h_{m}=(60 \pm 2) \mathrm{nm}$, at the scale of tip size, as determined by an AFM-based technique[15]. The elastic constant associated to the stretching is $B=$ $(240 \pm 10) \mathrm{mN} / \mathrm{m}$ [15] with $B=E h_{m} /\left(1-\sigma^{2}\right)$, where $\sigma$ and $E$ stand for the Poisson ratio and Young Modulus respectively. The resulting height $H$ and radius of curvature $R$ of the cross-section in a vertical plane (Fig. 1) are obtained by means of a camera (Nikon DXM1200) which images the sample from the side. Thus, our procedure results in an almost spherical (radius of curvature $R$ ) membrane, stretched over a circular frame, which encloses a chosen constant-volume of liquid.

A force $F$ is applied at the membrane center by means of a flexible micro-needle (Fig. 1, Top), whose tip is rounded to avoid damaging of the membrane. The radius of the bead tip is $\rho \simeq 11 \mu \mathrm{m}$ (Fig. 1, Bottom). In addition, in order to insure that only the bead touches the membrane, the needle tip is bent making almost a right angle with the membrane plane around the contact point. The micro-needle calibration is performed by recording the tip deflection due to the force applied by a AFM cantilever of known compliance. For the whole set of data reported below, the force $F=k_{n} \delta_{n}$, where the stiffness $k_{n}=(65 \pm 3) 10^{-3} \mathrm{~N} / \mathrm{m}\left(\delta_{n}\right.$ stands for the deflection of the needle along the normal to the surface). To obtain $\delta_{n}$ and the corresponding indentation, $\delta_{m}$, the membrane is imaged from the side. Good contrast in images is obtained by guiding light from a LASER inside the needle (Fig. 1, Bottom). The distance $\delta_{m}$ is obtained directly from the images whereas the deflection $\delta_{n}$ is obtained as the vertical deflection of the needle tip with respect to a reference line attached to the needle. Note that the total vertical displacement imposed to the needle writes $\delta_{m}+\delta_{n}$. Notice that a right choice of the microneedle stiffness ensures a

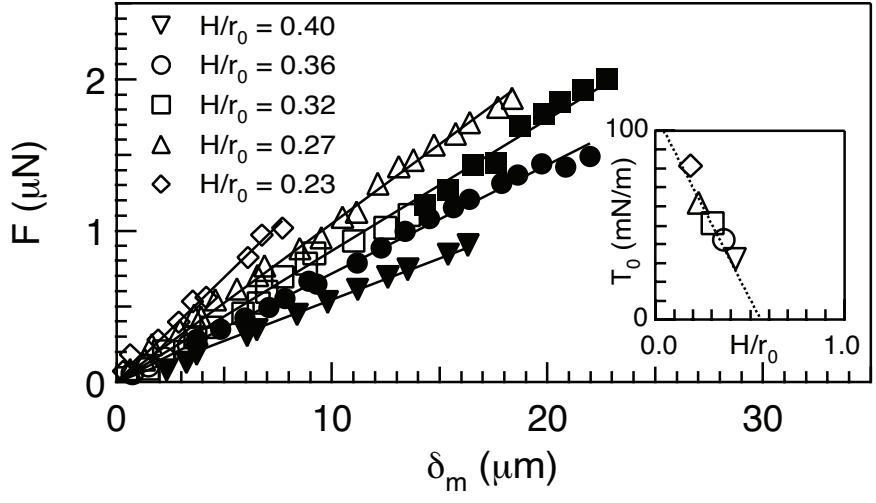

Fig. 2. Force $F$ vs. displacement $\delta_{m}$.

The force $F$ increases with $\delta_{m}$. The result holds true without (Open symbols) or with (Full symbols) wrinkles. Inset, the stiffness $k$ and, thus, the tension $T_{0}$ decrease with the initial height $H$ (or volume $V$ ).

membrane deflection that is of the same order than the tip deflection which, in turn optimizes the sensitivity of the force measurements. The sample is also imaged from the bottom by an optical microscope (Nikon Eclipse TE-300) and a high resolution SLR digital camera (Nikon D1X) using the Nomarsky contrast mode which makes possible both to determine the membrane features and to detect the wrinkles, even if the amplitude of the associated vertical displacement is small (Fig. 1, right panels).

\subsection{Force-displacement relation}

Qualitatively, when the membrane center is pushed downwards, a region of positive radial slope develops around the needle tip. The radius $r_{c}$ of this region is defined by the location of the circular apex, clearly visible as a brighter ring in the images from the bottom (Fig. 1, right panel a, dashed circle). The radius $r_{c}$ increases when the imposed displacement $\delta_{m}$ is increased and, depending on the preparation of the membrane, radial wrinkles are likely to appear above a given onset (Fig. 1, right panel b). Experimentally, $F$ is proportional to $\delta_{m}$ in a large range $\left(\delta_{m} \in[0,2 \rho]\right.$, Fig. 2). The conclusion interestingly holds true even when wrinkles form.

In the following, we estimate the relation between the stiffness $k$ and the initial tension of the membrane $T_{0}$ by calculating numerically the variation of the membrane surface energy which results from the indentation at constant inner volume $V$. In principle, the surface energy of the membrane has two contributions : a surface energy $\gamma$ (corresponding to the liquid-membrane interface) and the energy associated to the elastic modulus $B$ (the membrane being very thin, we neglect the energy associated with the bending). However, the surface tension $\gamma$ is expected to be small (at least much smaller than the surface tension associated with the air-PDMS liquid interface, $\gamma \simeq 23 \mathrm{mN} / \mathrm{m}$ ) as the liquid and the membrane are made of the same material. Thus, in a first step, we can neglect the contribution of the surface tension and assume that the initial 
tension of the membrane $T_{0}$ only originates by the elastic contribution (the initial stretching of the membrane is due to the preparation process). We shall see that the experimental value of $T_{0}$ is usually large enough for the latter assumption to be well satisfied. The main conceptual steps to achieve our numerical calculations are given below, a detailed description will be presented elsewhere. Our analysis is based on the minimization of the elastic free energy per unit surface, $\Gamma$, which only depends on the strain field $\underline{u}$ and the elastic constant $B$. The axissymmetric membrane is described by the radial $\delta r$ and the vertical $\delta z$ displacements which are calculated with respect to a membrane without tension. This means that the tension $T_{0}$ itself is accounted for by an initial isotropic stretching of the membrane. Thus, in any of the considered cases, either the flat or the spherical membrane, the shape of the indented membrane results from an initial deformation produced by a homogenous tension $T_{0}$, plus the additional deformation produced by the pushing tip. The calculation of the latter strain field, satisfying the conditions at both the pushing tip and the circular outer boundary, is the goal of the numerics. In a first approximation, the boundary condition at a spherical tip being difficult to account for, we consider instead a cylindrical tip, of radius $r_{t}$, and we mimic the indentation by a vertical displacement of the membrane in a circle of radius $r_{t}$. In practice, the Lagrangian is, $\mathcal{L}=\int_{S_{0}} \Gamma d S-\lambda \int_{S} d V$, where the first term is estimated by integrating over the initial shape of the membrane whereas the second term is estimated by integrating over its deformed shape. The differential equations that govern $\delta r$ and $\delta z$ are obtained from the variational transformation whose approximated solution are obtained by a shooting method. Starting from the center, where the action of the indenter is a vertical displacement of the membrane, i.e., $\delta r=0$ and $\delta z=-\delta_{m}$ in a circle of radius $r_{t}$, we look the correct matching at the outer boundary, where we consider that the indentation does not produce any additional deformation; $\delta r=0$ and $\delta z=0$ at $r=r_{0}$. The integration is performed with an adaptive step size control in the Runge Kutta integration method.

With the approximation above, the stiffness is found to satisfy,

$$
\frac{k}{T_{0}}=0.39+2 \pi\left(r_{t} / r_{0}\right)^{\beta},
$$

with $\beta=0.37 \pm 0.04$. Equation 1 holds true, to within $5 \%$, in a large range of radius of curvature $R$ and elastic modulus $B\left(\in\left[0,10 T_{0}\right]\right)$. Note that $k$ is thus found, in the elastic case, to increase slowly with $r_{t}$ [like $\left(r_{t} / r_{0}\right)^{\beta}$ and not like $\log \left(r_{t} / r_{0}\right)$ as found when the surface tension alone is taken into account] which explains why the forcedisplacement relation still remains linear in the case of a spherical indenter. Indeed, in this latter case, $r_{t}$ corresponds to the radius of the contact region between the tip and the membrane which rapidly reaches a value of the order of $\rho$ when $\delta_{m}$ is increased. Finally, we point out that a rather large imposed displacement, $\delta_{m}$, at the center does not necessarily induce large in-plane strains.
Experimentally, we observe that $k$ decreases when the initial volume $V$ (or equivalently $1 / R$ ) increases. However, according to Eq. 1, this dependance of $k$ on $V$ is not due to the change in the curvature $1 / R$ of the membrane. The dependance of $k$ on $V$ is due to the thermal dilation during the preparation process which leads to an initial tension $T_{0}$ that depends on the geometry of the droplet. Indeed, during the preparation, the fluid is heated in order to produce the polymerization of the free surface. After polymerization, the temperature and, thus, the surface area of the membrane without tension and the inner volume change. The thermal effects thus tend to change the surface to volume ratio and, as a consequence, to affect the membrane tension. In order to prove that the amplitude of the thermal effects is enough for accounting for the experimental changes in the membrane tension, we considered numerically the case of only a thermal contraction of the inner volume and estimated the resulting change in the tension $T_{0}$. Assuming that the polymerization leads to a reproducible initial tension $T_{0}^{*}$ and considering only a relative decrease $\theta$ of the entire fluid volume, we obtained that the tension $T_{0}$ of the membrane at the center should decrease according to $T_{0}=T_{0}^{*}-1.66 B \theta \frac{H}{r_{0}}$ in our experimental conditions. Experimentally, the tension is expected to vanish for $H / r_{0} \simeq 0.54$ (Fig. 2, inset) which permits to estimate the dilation $\theta$ that would account for the experimental variation of the tension. Taking into account the ratio $\frac{B}{T_{0}^{*}} \simeq 4$ [15], we obtain $\theta \simeq 30 \%$, which corresponds, the thermal expansion coefficient of the fluid being $3.510^{-4} K^{-1}$ [16], to a temperature-change of about $200 \mathrm{~K}$, which is a reasonable value considering that the fluid layer is thin and the temperature increase required for surface vulcanization is the same order. Thus, our analysis indicate that the membrane highly contract immediately after preparation. Note also that the thermal effects are likely to explain the large value of the tension $T_{0}$ (about $140 \mathrm{mN} / \mathrm{m}$ ) which is expected in the limit $H \rightarrow 0$.

Thus, indentation provides a reliable method for measuring the local tension $T_{0}$ of the membrane. The force is proportional to the displacement. The stiffness $k$ is proportional to the tension $T_{0}$, does not depend on the local curvature and only slowly increases with the size of the indenter. The result holds true even when the displacement is as large as the indenter diameter and when the membrane undergoes a wrinkling instability. However, we expect the curvature of the membrane to play a role when it compares with the typical size of the tip. Indeed, from the tip point of view, the membrane is flat as long as the radius of curvature of the membrane is much larger than the tip typical size. In additional experiments, to be published elsewhere, using latex membranes of $20 \mathrm{~cm}$ in diameter, inflated at a controlled pressure and indented by tips of large size, we have observed that the effect of membrane curvature remains weak. 

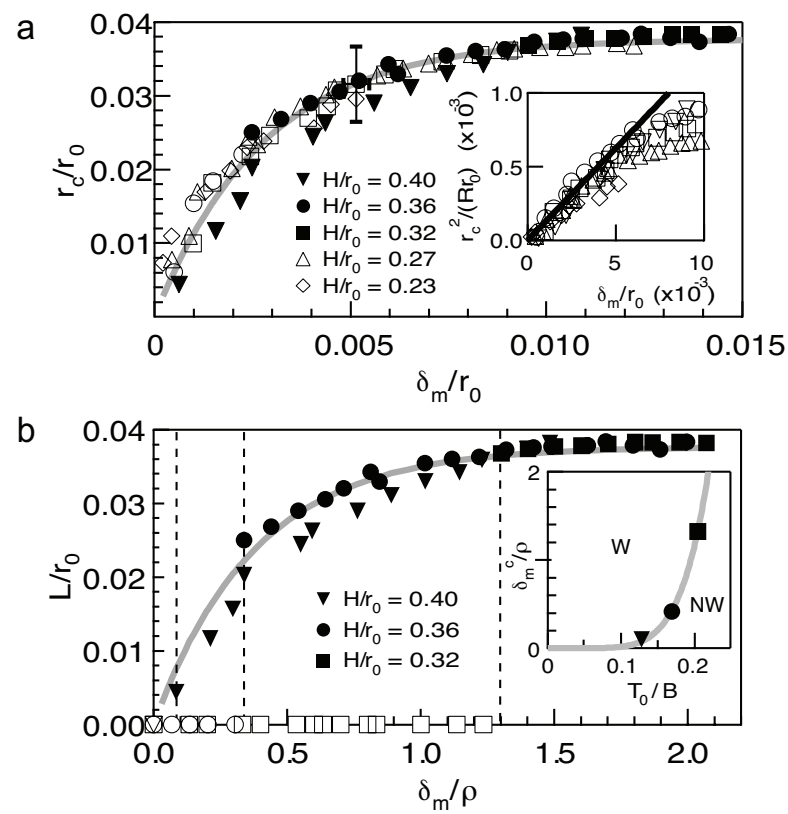

Fig. 3. Radius $r_{c}$ vs. displacement $\delta_{m}$.

a) $r_{c}$ increases almost exponentially (grey line) with $\delta_{m}$. The result holds true without (Open symbols) or with (Full symbols) wrinkle pattern (The typical error bar is displayed on a single point). Inset: $r_{c}^{2} / R$ vs $\delta_{m}$ shows that $r_{c} \propto \sqrt{R \delta_{m}}$ for small $\delta_{m}$, with an initial slope $\approx 1 / 8$. b) $L$ increases almost exponentially with $\delta_{m}$. The grey line is the exponential fit displayed in panel (a). Arrows mark the transitions to wrinkling and $L$ is taken equal to zero below these thresholds. Inset: $\delta_{m}^{c}$, drastically increases with $T_{0}$. Wrinkles $(\mathrm{W})$ and No Wrinkles (NW) regions.

\subsection{Membrane shape and instability}

We observe that the radius of the concave part around the tip, $r_{c}$, increases almost exponentially with $\delta_{m}$ (Fig. 3a),

$$
r_{c}=r_{c}^{\max }\left[1-\exp \left(-\frac{\delta_{m}}{\delta_{m}^{t y p}}\right)\right]
$$

and the appearance of wrinkles does not alter significantly the dependance of $r_{c}$ on $\delta_{m}$. The radius $r_{c}$ only slightly depends on the initial height $H$ and, thus, on the tension $T_{0}$ in the whole experimental range.

From the exponential interpolation of the data, we determine the characteristic deflection $\delta_{m}^{t y p} \simeq(2.8 \pm 0.1) \times$ $10^{-3} r_{0}$ and the asymptotic value $r_{c}^{\max } \simeq(38 \pm 1) \times$ $10^{-3} r_{0}$. The theoretical analysis as well as the numerical results suggest that, in the limit of small $\delta_{m}, r_{c} \propto \sqrt{R \delta_{m}}$. In the inset of the figure $3 \mathrm{a}$, we report $r_{c}^{2} / R$ as a function of $\delta_{m}$ and obtain experimentally $r_{c} \simeq \sqrt{R \delta_{m} / 8}$. Note however that the numerical results, that involve equations that are only valid in the limit of small strains, fail in accounting for the exponential growth of $r_{c}$ when the displacement $\delta_{m}$ is further increased (typically, when $\delta_{m} \simeq 5 \times 10^{-3} r_{0}$ which corresponds to $\delta_{m} \simeq 1.5 \rho$, the vertical displacement being thus larger than the radius of the pushing tip).

If $T_{0}$ is small enough and $\delta_{m}$ is increased above the critical value, $\delta_{m}^{c}$, the membrane is subjected to an ax- ial wrinkling instability (Fig. 1, right panel b). We note that wrinkles extend to the border of the concave region, i.e. the length of the wrinkle, $L$, almost equals the radius $r_{c}$ (Fig. 3b). The most interesting result lies in the fact that the critical displacement $\delta_{m}^{c}$ drastically depends on the tension $T_{0}$ (Fig. $3 \mathrm{~b}$, inset). For small $T_{0}$, the transition is nearly supercritical as $\delta_{m}^{c}$ almost vanishes. On the contrary, for slightly larger $T_{0}$, the instability is subcritical as $\delta_{m}^{c}$ takes a finite value: the membrane is subjected to a wrinkling instability provided that the imposed displacement is larger than a given, finite, value and, at the transition, the wrinkle length jumps to a finite value $\left[L=r\left(\delta_{m}^{c}\right)\right.$, Fig. $3 \mathrm{~b}$, circles and squares $]$. If $T_{0}$ is initially too large, wrinkles are no longer observed.

Results above can be understood considering that the membrane instability requires that the orthoradial tension becomes negative (compression) at regions where wrinkles are observed. The variation of the tension in the orthoradial direction at the distance $r$ from the center, $\sigma_{\perp}$, is about $B \frac{\delta r}{r}$ where $\delta r$ denotes the displacement of the membrane toward the center due the action of the tip so that $\sigma_{\perp} \sim T_{0}+B \frac{\delta r}{r}$. Due to the indentation, the membrane is locally pulled toward the center and we can estimate, assuming that the membrane is wrapping around the pushing tip, $\delta r \sim r_{t}(\sin \alpha-\alpha)$ at the distance $r_{t} \alpha$ from the center. The angle $\alpha$ stands for the angle that the membrane makes with the horizontal at the contact with the indenter. Even expected to mainly depend on the ratio $\delta_{m} / \rho$, the displacement $\delta r$ can hardly be estimated without solving the entire problem. However, the local-strain is estimated to never exceed $1-\frac{2}{\pi} \simeq 0.36$ when the displacement $\delta_{m}$ is increased. Accordingly, the membrane should be stable as long as $T_{0}>0.36 \mathrm{~B}$. Taking into account the value of the elastic modulus $B$, we thus predict, in acceptable agreement with the experimental results, that no wrinkle can form for a tension $T_{0}$ typically larger than $86 \mathrm{mN} / \mathrm{m}$. Finally, even if such a behavior cannot be accounted by the description of the system in the small displacement limit, we would like to mention that the interpolation of the experimental data leads to $\delta_{m}^{c}=\rho\left(a T_{0} / B\right)^{6}$, with $a=(5.0 \pm 0.2)$ for $B=(240 \pm 10) \mathrm{mN} / \mathrm{m}$ (Fig. $3 \mathrm{~b}$, inset). Although this latter relation would deserve further theoretical effort, the constant $a$ is a universal constant for the problem as long as the radius of the frame keeps much larger than the other lengths in the system. Thus, our result can be useful for the assessment of the modulus $B$ in similar systems.

\section{Conclusion}

We demonstrated experimentally that the measurement of the stiffness while pushing, with the help of a microneedle, a curved membrane enclosing a constant volume provides information about the tension $T_{0}$ of the membrane (or equivalently on the inner overpressure $\Delta P$, provided the radius of curvature is known) and not about the elastic constant $B$. Such stiffness measurements hold even in the presence of wrinkles. Wrinkling instability occurs when 
the membrane tension $T_{0}$ compares with the elastic modulus $B$, which provides an alternative method for measuring $B$ in small sized systems. For instance for capsules, made of a polymer network and inflated by osmotic pressure[7], one could decrease the osmotic pressure down to values at whose wrinkles develop. By reporting the behavior of the critical displacement $\delta_{m}^{c}$ as a function of $\Delta P$, which is simply related to $T_{0}$, the elastic modulus $B$ would be obtained.

In summary, our study shows that, the force - displacement relation in the linear regime, for an indentation type measurement, only provides information about initial tension of the membrane. The assessment of elastic modulus $\mathrm{B}$, can be achieved either from a nonlinear regime as in the case of reference [15] or by examination of the onset of the wrinkling pattern as in the present manuscript.

This research was supported by Conicyt-Chile under Anillo project No ACT-95.

\section{References}

1. A. K. Harris, P. Wild and D. Stopak, Science 28, (1980) 177.

2. E. Cerda, J. Biomech. 38(8), (2005) 1598.

3. K. Burton and D. L. Taylor, Nature 393, (1998) 150.

4. K. D. Hobart, J. Electron. Mater. 30(7), (2001) 797.

5. P. A. O'Connell and G. B. McKenna, Eur. Phys. J. E 20, (2006) 143.

6. E. P. Chan and A. J. Crosby, Advanced Materials, 18(24), (2006) 3238.

7. Vernita D. Gordon, Xi Chen, John W. Hutchinson, Andreas R. Bausch, Manuel Marquez and David A. Weitz, J. Am. Chem. Soc. 126(43), (2004) 14121.

8. Stefan J. Pastine, David Okawa, Alex Zettl and Jean M. J. Frchet, J. Am. Chem. Soc. 131(38), (2009) 13586.

9. M. W. Keller and N. R. Sottos, Experimental Mechanics 46(6), (2006) 725 .

10. E. Cerda, K. Ravi-Chandar and L. Mahadevan, Nature 419, (2002) 579.

11. E. Cerda and L. Mahadevan, Phys. Rev. Lett. 90, (2003) 1.

12. J-Ch. Géminard, R. Bernal and F. Melo, Eur. Phys. J. E 15, (2004) 117.

13. J. Huang, M. Juszkiewicz, W. H. de Jeu, E. Cerda, T. Emrick, N. Menon and T. P. Russell, Science 317, (2007) 650.

14. Christopher M. Stafford, Christopher Harrison, Kathryn L. Beers, Alamgir Karim, Eric J. Amis, Mark R. VanLandingham, Ho-Cheol Kim, Willi Volksen, Robert D. Miller and Eva E. Simonyi, Nature Materials 3, (2004) 545.

15. R. Bernal, Ch. Tassius, J-Ch. Géminard, R. Bernal and F. Melo, Appl. Phys. Lett., 90, (2007) 063903.

16. M. Li, S. Xu, and E. Kumacheva, Macromolecules $\mathbf{3 3}$, (2000) 4972. 